\author{
RECENT DEVELOPMENTS \\ IN FUNCTIONAL EQUATIONS AND INEQUALITIES \\ BANACH CENTER PUBLICATIONS, VOLUME 99 \\ INSTITUTE OF MATHEMATICS \\ POLISH ACADEMY OF SCIENCES \\ WARSZAWA 2013
}

\title{
MAREK KUCZMA
}

\author{
KAROL BARON \\ Institute of Mathematics, University of Silesia \\ Bankowa 14, 40-007 Katowice, Poland \\ E-mail:baron@us.edu.pl
}

\begin{abstract}
The scientific output of Marek Kuczma consists of 179 papers published in the years 1958-1993 and three books still used and quoted. Professor Marek Kuczma created and developed the theory of iterative functional equations but his name is also connected to important results on functional equations in several variables, in particular on Cauchy's equation and Jensen's inequality. In fact Marek Kuczma has founded a mathematical school: he supervised 13 Ph.D. dissertations, 10 his students have already their habilitation and 6 of them became full professors. The paper provides more information about the great teacher and some results of the outstanding mathematician.
\end{abstract}

Marek Kuczma was born on October 10, 1935, in Katowice (Poland). There he got the secondary school certificate on June 9, 1952. In the years 1952-1956 he studied mathematics at the Jagiellonian University.

1. Degrees. On June 25, 1956, he got M.Sc. and on January 19, 1961, the Ph.D., both from the Jagiellonian University. The Ph.D. dissertation, entitled $O$ pewnym równaniu funkcyjnym pierwszego rzeddu (On a functional equation of the first order) was supervised by Professor Stanisław Gołąb. His habilitation at the Jagiellonian University on October 17, 1963, was based on the dissertation [12. The State Council of Poland conferred him the titles of extraordinary and ordinary Professor of Mathematics on June 18, 1969, and May 21, 1973, respectively.

2. Academic. From January 1, 1956, to February 28, 1962, Marek Kuczma worked at the AGH University of Science and Technology (Chair of Mathematics), and from

2010 Mathematics Subject Classification: Primary 01A70; Secondary 39B12, 39B22, 39B32, 39B52, 39B62, 26B25, 26B40, 30D05, 51M04, 60J80.

Key words and phrases: Marek Kuczma, his descendants, short CV and some results on functional equations.

The paper is in final form and no version of it will be published elsewhere. 
March 1, 1962, to November 30, 1966, at the Jagiellonian University (Chair of Geometry). Since October 1963 he was deputy head of the Section of Mathematics for the Katowice Branch affairs and then, since May 1964, vice-dean of the Faculty of Mathematics, Physics and Chemistry for the Katowice Branch affairs. On December 1, 1966, he was appointed head of the Chair of Functional Equations in the Katowice Branch of the Jagiellonian University and was vice-dean of the Faculty of Mathematics, Physics and Chemistry of the Jagiellonian University for the Katowice Branch affairs up to June 7, 1968.

On June 8, 1968, the Silesian University of Katowice was established and Marek Kuczma had been a professor (since June 1973 full professor) of this University from the very beginning till his death on June 13, 1991, being the head of the Chair of Functional Equations until August 31, 1987, and the head of the Department of Mathematics until September 30, 1973.

From October 1, 1966, to September 30, 1968 he was also working at the Institute of Mathematics of the Polish Academy of Sciences.

3. Editorial. Professor Marek Kuczma was a member of the editorial board of Aequationes Mathematicae from the foundation of the journal in 1968.

4. Awards. He got twice (in 1968 and 1971) the Prize of the Minister of Higher Education and was honoured with the Gold Cross of Merit (in 1970) and with the Officer's Cross of the Order Polonia Restituta (in 1990).

5. Students. Professor Kuczma supervised 13 Ph.D. dissertations, 10 of his students have already their habilitations and 6 of them became full professors. The list of students in chronological order follows (one star indicates the year of habilitation and two stars the year in which the person became full professor): Dobiesław Brydak (1966, Jagiellonian University; 1977*), Jerzy Kordylewski (1968, Jagiellonian University), Wilhelmina Smajdor (1968, Polish Academy of Sciences; 1987*), Andrzej Smajdor (1969, Jagiellonian University; 1987* 2001**), Janusz Matkowski (1970, Jagiellonian University; 1975*, 1983**), Roman Ger (1971, Silesian University; 1976*, 1990**), Stefan Czerwik (1971, Jagiellonian University; 1983*), Małgorzata Rozmus-Chmura (1972, Jagiellonian University), Karol Baron (1974, Silesian University; 1980*, 1996**), Marek Cezary Zdun (1974, Silesian University; 1980* $1997^{* *}$ ), Joanna Ger (1975, Silesian University), Józef Drewniak (1975, Silesian University; 1990*), Maciej Sablik (1980, Silesian University; 1997*, 2011**). The following seven scientific grandchildren of Professor Marek Kuczma have already their habilitations: Kazimierz Nikodem $\left(1991^{*}, 2001^{* *}\right)$, Witold Jarczyk (1993*, 2010**), Roman Badora $\left(2007^{*}\right)$, Janusz Morawiec $\left(2009^{*}\right)$, Michał Baczyński $\left(2010^{*}\right)$, Justyna Sikorska $\left(2011^{*}\right)$, Krzysztof Ciepliński $\left(2012^{*}\right)$. Szymon Wąsowicz $\left(2011^{*}\right)$ is up to now the only scientific great-grandchild of Professor Kuczma having already his habilitation.

6. Reports. Professor Marek Kuczma evaluated 26 Ph.D. theses and was a referee in 14 habilitation procedures, in 15 professorship procedures and in 2 academy fellowship procedures. 
7. Books. Marek Kuczma wrote two monographs on functional equations in a single variable [15, 24] and one on functional equations in several variables [21]. All these three books are still used and quoted.

8. Papers. Within the years 1958-1993 Marek Kuczma published 179 papers. They are listed in the obituary [7] by R. Ger.

\section{Selected results}

1. Let us start with continuous solutions of the functional equation

$$
\varphi(f(x))=g(x) \varphi(x)
$$

under the following assumptions on the given functions $f$ and $g$ :

The function $f$ maps continuously a real interval $I$ into itself in such a manner that the inequalities

$$
0<\frac{f(x)-\xi}{x-\xi}<1
$$

hold with a $\xi \in I$ for every $x \in I \backslash\{\xi\}$. The function $g: I \rightarrow \mathbb{R}$ is continuous and $g(x) \neq 0$ for every $x \in I \backslash\{\xi\}$.

Studying in [12] the $C^{1}$-solutions $\varphi$ of the Schröder equation

$$
\varphi(f(x))=s \varphi(x)
$$

and continuous solutions of the equation for $\varphi^{\prime}$ :

$$
\Phi(f(x))=\frac{s}{f^{\prime}(x)} \Phi(x),
$$

Professor Marek Kuczma distinguished (in full generality jointly with Bogdan Choczewski in [4]) the following three cases:

(A) The sequence $\left(G_{n}\right)$ defined by

$$
G_{n}=\prod_{j=0}^{n-1} g \circ f^{j}
$$

converges pointwise to a continuous map of $I$ into $\mathbb{R} \backslash\{0\}$.

(B) The sequence $\left(G_{n}\right)$ converges to zero uniformly on a subinterval of $I$.

(C) Neither (A), nor (B) occurs.

In case $(\mathrm{A})$ a function $\varphi: I \rightarrow \mathbb{R}$ is a continuous solution of (1) if and only if there exists a real constant $c$ such that

$$
\varphi(x)=\frac{c}{\lim _{n \rightarrow \infty} G_{n}(x)} \quad \text { for every } x \in I .
$$

In case $(\mathrm{C})$ the only continuous solution $\varphi: I \rightarrow \mathbb{R}$ of $(1)$ is the zero function.

What about case (B)? Assume additionally that $f$ is strictly increasing. (Concerning this additional assumption consult [1] by J. Kalinowski and M. Kuczma.)

Then, in case (B), there exists a subinterval $J$ of I such that each continuous function $\varphi_{0}: J \rightarrow \mathbb{R}$ has an extension to a continuous solution $\varphi: I \rightarrow \mathbb{R}$ of $(1)$.

How to get all the continuous solutions in that case? Let me only mention that it was an open problem for 14 years eventually solved by Marek Kuczma in [20]. 
2. In the above mentioned dissertation 12 Professor Marek Kuczma studied also convex solutions of the Schröder equation (2). In [13] he returned to this problem considering solutions $\varphi$ such that $\frac{\varphi(x)}{x}$ is a monotonic function. We present the result:

Assume that the real interval $I$ has the form $[0, a]$ or $[0, a)$ where $a$ is a positive number, and let the function $f: I \rightarrow I$ be continuous, increasing, differentiable at zero with

$$
s:=f^{\prime}(0) \in(0,1)
$$

and such that

$$
0<f(x)<x \quad \text { for every } x \in I \backslash\{0\} .
$$

Fix arbitrarily an $x_{0} \in I \backslash\{0\}$.

If $\varphi: I \rightarrow \mathbb{R}$ is a non-zero solution of (2) such that $\frac{\varphi(x)}{x}$ is a monotonic function, then there exists a real constant $c$ such that

$$
\varphi(x)=c \lim _{n \rightarrow \infty} \frac{f^{n}(x)}{f^{n}\left(x_{0}\right)} \quad \text { for every } x \in I .
$$

Moreover, if $\frac{f(x)}{x}$ is a monotonic function, then for every $x \in I$ the sequence $\left(\frac{f^{n}(x)}{f^{n}\left(x_{0}\right)}\right)$ converges to a positive and finite limit, and the function $\varphi_{0}: I \rightarrow \mathbb{R}$ defined by

$$
\varphi_{0}(x)=\lim _{n \rightarrow \infty} \frac{f^{n}(x)}{f^{n}\left(x_{0}\right)}
$$

is a continuous solution of (2) and $\frac{\varphi_{0}(x)}{x}$ is a monotonic function. If $f$ is convex (resp. concave), then $\varphi_{0}$ is convex (resp. concave) and strictly increasing.

This theorem has been applied by C. R. Heathcote, E. Seneta and D. Vere-Jones [10] to get the limit of the sequence of probabilities $\operatorname{Prob}\left(Z_{n}=j \mid n<T<\infty\right)$ for the Galton-Watson process $\left(Z_{n}\right)$ such that

$$
\operatorname{Prob}\left(Z_{1}=0\right)>0 \quad \text { and } \operatorname{Prob}\left(Z_{1}=j\right)<1 \quad \text { for } j \in \mathbb{N} \cup\{0\}, \quad E Z_{1} \neq 1,
$$

where $T$ denotes the extinction time:

$$
T= \begin{cases}\min \left\{n \in \mathbb{N}: Z_{n}=0\right\}, & \text { if } Z_{n}=0 \text { for some } n \in \mathbb{N}, \\ +\infty, & \text { if } Z_{n} \neq 0 \text { for every } n \in \mathbb{N} .\end{cases}
$$

To finish this part of selected results let us only mention that also Professor Marek Kuczma's theorem on convex solutions of the Abel equation

$$
\varphi(f(x))=\varphi(x)+1
$$

(proved in [14) has been applied in the theory of branching processes (by E. Seneta in [26]).

3. Professor Marek Kuczma liked convex functions very much indeed. They are present in his research from the very beginning up to the end. In [18] he obtained a perfect analogue of N. G. de Bruijn's result on almost additive functions:

$$
\begin{aligned}
& \text { If } f:(a, b) \rightarrow \mathbb{R} \text { satisfies } \\
& \qquad f\left(\frac{x+y}{2}\right) \leq \frac{f(x)+f(y)}{2} \quad \text { for a.e. pair }(x, y) \in(a, b)^{2},
\end{aligned}
$$


then the function $g:(a, b) \rightarrow \mathbb{R}$ given by

$$
g(x)=\inf \operatorname{ess}\left\{\frac{1}{2}(f(x+h)+f(x-h)): x-h, x+h \in(a, b)\right\}
$$

is the unique Jensen convex function on $(a, b)$ such that

$$
g(x)=f(x) \quad \text { for a.e. } x \in(a, b) .
$$

Concerning an abstract version of this theorem see Section 17.8 in [21.

An interesting stability result on Jensen convexity is contained in [22] by Marek Kuczma:

Assume that $f$ is a Jensen convex function defined in an open subset $D$ of a linear topological space $X$. If there is a set $T \subset D$ such that

(i) every Jensen convex function upper bounded on $T$ is continuous,

(ii) $T$ is symmetric with respect to a point,

(iii) $\inf \left\{f\left(\frac{x+y}{2}\right)-\frac{f(x)+f(y)}{2}: x, y \in T\right\}>-\infty$,

then

$$
f(x)=F(x)+a(x) \quad \text { for every } x \in D
$$

with an additive $a: X \rightarrow \mathbb{R}$ and a continuous convex function $F: D \rightarrow \mathbb{R}$.

In two lemmas Professor Kuczma proved there also that:

(i) any second category Baire subset of a linear topological space contains a second category Baire set that is symmetric with respect to a point,

(ii) any Lebesgue measurable set of positive measure contains a Lebesgue measurable set of positive measure that is symmetric with respect to a point.

4. Each solution $f$ of the Cauchy equation with values in a linear space satisfies

$$
f(x+y) \in \operatorname{Lin}\{f(x), f(y)\}
$$

i.e.,

$$
f(x+y)=\varphi(x, y) f(x)+\psi(x, y) f(y)
$$

with some scalar functions $\varphi$ and $\psi$. J. Aczél, B. Forte and C. T. Ng have solved this equation in [1] under the conditions that $f: \mathbb{R}^{N} \rightarrow \mathbb{R}^{N}, \varphi, \psi: \mathbb{R}^{2 N} \rightarrow \mathbb{R}$ are continuous, $N \geq 3$ and

$$
\text { if } x, y \in \mathbb{R}^{N} \text { are linearly independent, then so are also } f(x), f(y) \text {. }
$$

Functions $\varphi, \psi$ do not appear in the geometrical formulation of (3) and their continuity does not follow readily from the continuity of $f$. In [19] Professor Kuczma removed this additional regularity assumption. His results read as follows.

If $N \geq 3$, then a continuous $f: \mathbb{R}^{N} \rightarrow \mathbb{R}^{N}$ satisfies (3) with some $\varphi, \psi: \mathbb{R}^{2 N} \rightarrow \mathbb{R}$ and (4) if and only if

$$
f(x)=\gamma(x) A x \quad \text { for every } x \in \mathbb{R}^{N}
$$

with a nonsingular $N \times N$ matrix $A$ and $a \gamma: \mathbb{R}^{N} \rightarrow(0, \infty)$ which is continuous except, possibly, at the origin and

$$
\lim _{x \rightarrow 0} \gamma(x) x=0
$$


If $f: \mathbb{R}^{N} \rightarrow \mathbb{R}^{N}$ has form (5) where $A$ is a nonsingular $N \times N$ matrix and $\gamma: \mathbb{R}^{N} \rightarrow$ $\mathbb{R} \backslash\{0\}$, then $\varphi, \psi: \mathbb{R}^{2 N} \rightarrow \mathbb{R}$ satisfy (3) if and only if

$$
\varphi(x, y)=\frac{\gamma(x+y)+\alpha(x, y)}{\gamma(x)}, \quad \psi(x, y)=\frac{\gamma(x+y)+\beta(x, y)}{\gamma(y)}
$$

for $x, y \in \mathbb{R}^{N}$ with some $\alpha, \beta: \mathbb{R}^{2 N} \rightarrow \mathbb{R}$ such that

$$
\alpha(x, y) x+\beta(x, y) y=0 \quad \text { for every } x, y \in \mathbb{R}^{N} .
$$

5. Given $p: \mathbb{R} \rightarrow \mathbb{R}$ consider the family $M_{p}$ of all straight lines in $\mathbb{R}^{2}$ which are parallel to the $y$-axis and of all curves of the form

$$
y=p(x+\alpha)+\beta
$$

with $\alpha, \beta \in \mathbb{R}$. Based on the solution of a functional equation related to the functional equation of Cauchy Marek Kuczma jointly with Vance Faber and Jan Mycielski proved in [5] the following result on plane geometry.

If there exists a continuous bijection of $\mathbb{R}^{2}$ onto itself which induces a map of the family of all straight lines onto $M_{p}$, then $p$ must be a polynomial of degree 2 .

This theorem was shown in [9] by B. Grünbaum and J. Mycielski under the additional condition that $p$ is differentiable.

6. In 23] Professor Kuczma considered the equation

$$
\varphi(z) \overline{\varphi\left(-\frac{1}{\bar{z}}\right)}=-1
$$

which comes from astrophysics. He proved there that

(i) the general solution $\varphi: \mathbb{C} \backslash\{0\} \rightarrow \mathbb{C} \backslash\{0\}$ of $(6)$ is given by

$$
\varphi(z)=\frac{z f(z)}{\overline{f\left(-\frac{1}{\bar{z}}\right)}} \quad \text { for every } z \in \mathbb{C} \backslash\{0\},
$$

where $f: \mathbb{C} \backslash\{0\} \rightarrow \mathbb{C} \backslash\{0\}$ is an arbitrary function;

(ii) if $\varphi: \mathbb{C} \backslash\{0\} \rightarrow \mathbb{C} \backslash\{0\}$ is a solution of $(6)$ that is analytic on $\mathbb{C} \backslash\{0\}$ and has a removable singularity or a pole at the origin, then there exists an integer $k$ and $a$ complex constant $\eta$ with $|\eta|=1$ such that

$$
\varphi(z)=\eta z^{2 k+1} \quad \text { for every } z \in \mathbb{C} \backslash\{0\} .
$$

7. The last part of selected results obtained by Professor Kuczma is connected with the following two very interesting problems posed by Z. Moszner in [25].

Given $N \in \mathbb{N}$ and $r \in \mathbb{N} \cup\{\infty\}$ put

$$
D_{N}^{r}=\left\{f: \mathbb{R}^{N} \rightarrow \mathbb{R}^{N} \mid f \text { is of the class } C^{r} \text { and } \operatorname{Det} f^{\prime}(x)>0 \text { for } x \in \mathbb{R}^{N}\right\} .
$$

Problem 1. For every $f \in D_{N}^{r}$ does there exist $a \varphi \in D_{N}^{r}$ such that

$$
\varphi^{2}=f ?
$$


Problem 2. For every $f \in D_{N}^{r}$ does there exist an $n \in \mathbb{N}$ and $\varphi_{1}, \ldots, \varphi_{n} \in D_{N}^{r}$ such that

$$
f=\varphi_{1}^{2} \circ \ldots \circ \varphi_{n}^{2} ?
$$

In [16] Professor Marek Kuczma:

(i) constructed an $f \in D_{2}^{\infty}$ such that equation (7) has no solution at all;

(ii) proved that

if $r \in \mathbb{N} \cup\{\infty\}$ and $f \in D_{1}^{r}$ has no fixed point, then (7) has a solution $\varphi \in D_{1}^{r}$;

(iii) constructed examples showing that in the above theorem the assumption $f(x) \neq x$ for every $x \in \mathbb{R}$ is essential (and, consequently, showed that Problem 1 has negative solution also in the one-dimensional case).

Regarding Problem 2 he proved in [17] that

for every $r \in \mathbb{N} \cup\{\infty\}$ and for every $f \in D_{1}^{r}$ there exist $\varphi_{1}, \ldots, \varphi_{4} \in D_{1}^{r}$ such that

$$
f=\varphi_{1}^{2} \circ \ldots \circ \varphi_{4}^{2} \text {. }
$$

For $N>1$ Problem 2 is still open.

At the age of 45, in August 1980, Professor Marek Kuczma suffered a second brain stroke and became seriously paralysed. But we just saw some of his results obtained after this year. Professor Kuczma was able to continue his scientific activity (he wrote at that time 26 articles and was intensively involved in preparation of two books) not only thanks to his will power but, above all, due to the care, angelic patience and devotion of his wife Krystyna. They were married in 1963. Mrs. Krystyna Kuczma died on December 30, 1993.

Acknowledgments. I cordially thank Professors Janusz Brzdęk and Roman Ger for inviting me to give a talk about Marek Kuczma during the 14th International Conference on Functional Equations and Inequalities and to include it in this volume. Preparing the talk I used [3] by B. Choczewski, 6, 7] by R. Ger, and [2]. In those four articles as well as in [8] by R. Ger some further information and results of Professor Marek Kuczma may be found.

\section{References}

[1] J. Aczél, B. Forte, C. T. Ng, On a triangular functional equation and some applications, in particular to the generalized theory of information, Aequationes Math. 11 (1974), 11-30.

[2] K. Baron, M. Kuczma's papers on iterative functional equations, in: Selected Topics in Functional Equations and Iteration Theory (Graz, 1991), Grazer Math. Ber. 316, KarlFranzens-Univ. Graz, Graz, 1992, 1-6.

[3] B. Choczewski, Papers of Marek Kuczma written in the last decade of his life, in: Selected Topics in Functional Equations and Iteration Theory (Graz, 1991), Grazer Math. Ber. 316, Karl-Franzens-Univ. Graz, Graz, 1992, 7-16. 
[4] B. Choczewski, M. Kuczma, On the "indeterminate case" in the theory of a linear functional equation, Fund. Math. 58 (1966), 163-175.

[5] V. Faber, M. Kuczma, J. Mycielski, Some models of plane geometries and a functional equation, Colloq. Math. 62 (1991), 279-281.

[6] R. Ger, M. Kuczma's papers on functional equations in several variables, in: Selected Topics in Functional Equations and Iteration Theory (Graz, 1991), Grazer Math. Ber. 316, Karl-Franzens-Univ. Graz, Graz, 1992, 17-28.

[7] R. Ger, Marek Kuczma, 1935-1991, Aequationes Math. 44 (1992), 1-10.

[8] R. Ger, Functional equations and inequalities, in: Half a century of mathematics in Upper Silesia, Pr. Nauk. Uniw. Śl. Katow. 2196, Wydawn. Uniw. Śląskiego, Katowice, 2003, 223-251 (in Polish).

[9] B. Grünbaum, J. Mycielski, Some models of plane geometries, Amer. Math. Monthly 97 (1990), 839-846.

[10] C. R. Heathcote, E. Seneta, D. Vere-Jones, A refinement of two theorems in the theory of branching processes, Teor. Verojatnost. i Primenen. 12 (1967), 341-346; English transl.: Theor. Probability Appl. 12 (1967), 297-301.

[11] J. Kalinowski, M. Kuczma, Some remarks on continuous solutions of a functional equation, Uniw. Śląski w Katowicach Prace Nauk.-Prace Mat. 9 (1979), 64-73.

[12] M. Kuczma, On the Schröder equation, Rozprawy Mat. 34 (1963).

[13] M. Kuczma, Note on Schröder's functional equation, J. Austral. Math. Soc. 4 (1964), 149-151.

[14] M. Kuczma, On convex solutions of Abel's functional equation, Bull. Acad. Polon. Sci. Sér. Sci. Math. Astronom. Phys. 13 (1965), 645-648.

[15] M. Kuczma, Functional Equations in a Single Variable, Monografie Matematyczne 46, Państwowe Wydawnictwo Naukowe, Warszawa, 1968.

[16] M. Kuczma, Fractional iteration of differentiable functions, Ann. Polon. Math. 22 (1969/70), 217-227.

[17] M. Kuczma, On squares of differentiable functions, Ann. Polon. Math. 22 (1969/70), 229-237.

[18] M. Kuczma, Almost convex functions, Colloq. Math. 21 (1970), 279-284.

[19] M. Kuczma, On mappings preserving linear dependence and independence, Uniw. Śląski w Katowicach Prace Nauk.-Prace Mat. 7 (1977), 29-49.

[20] M. Kuczma, General continuous solution of a linear homogeneous functional equation, Ann. Polon. Math. 35 (1977/78), 21-25.

[21] M. Kuczma, An Introduction to the Theory of Functional Equations and Inequalities. Cauchy's Equation and Jensen's Inequality, Uniwersytet Śląski, Katowice; Państwowe Wydawnictwo Naukowe, Warszawa, 1985; Second edition, edited and with a preface by A. Gilányi, Birkhäuser, Basel, 2009.

[22] M. Kuczma, On a result of Z. Kominek, Rad. Mat. 3 (1987), 307-315.

[23] M. Kuczma, On a functional equation occurring in astrophysics, Math. Pannon. 3 (1992), $17-27$.

[24] M. Kuczma, B. Choczewski, R. Ger, Iterative Functional Equations, Encyclopedia Math. Appl. 32, Cambridge Univ. Press, Cambridge, 1990.

[25] Z. Moszner, P.2, Aequationes Math. 1 (1968), 150.

[26] E. Seneta, The Galton-Watson process with mean one, J. Appl. Probability 4 (1967), $489-495$. 